\title{
Thracker - Using Capacitive Sensing for Gesture Recognition
}

\author{
Raphael Wimmer, Paul Holleis, Matthias Kranz, Albrecht Schmidt \\ Research Group Embedded Interaction \\ University of Munich \\ Amalienstrasse 17, 80333 Munich, Germany \\ \{raphael, albrecht, paul, matthias\}@ hcilab.org
}

\begin{abstract}
In this paper we present Thracker - a low-cost and robust hardware to track hand gestures in front of a screen or small-scale active spaces like public displays or posters. Thracker uses capacitive sensing for tracking user input. Thracker allows for entire new interaction modes like picking and dropping an object on the screen with the hand. We present a fully working prototype and a short user study to confirm our findings.
\end{abstract}

\section{Introduction}

Novel input technologies have the potential of changing the way we interact with computer systems. With advances in different areas related to information and communication technologies, the term computer includes traditional personal computers but more importantly relates to appliances and embedded interactive systems. The use of appliances beyond the desktop has spread into users' daily lives. Interaction with computational systems has become the norm; whether we make a phone call, buy a ticket, or use an elevator we are interacting with a computer. The requirements for input of such embedded interactive systems differ greatly between appliances and are most often very different from traditional desktop computers. In some cases the input required is only a selection (e.g. one out of 12 in an elevator control) whereas for other systems a highly precise control with real-time response may be required.

We investigate the use of capacitive sensing as alternative input mode for appliances. We particularly look at two dimensional pointing and gestures as input primitives. Depending on the form factor of the appliance, required input dimensions and precision, size and weight, learnability and acceptable cost, the use of capacitive sensing may be an interesting alternative to conventional techniques. In this paper we explore the potential of capacitive sensing for $2 \mathrm{D}$ tracking and gesture input; the system is called Thracker.
Figure 1 shows an example image viewer used for navigating large $\mathrm{X}$-ray images.

Basic input parameters supported by Thracker are absolute coordinates (like a touch screen) and relative movements (mouse-like) as well as simple gestures. Gestures can be single points moving over time or multiple points moving simultaneously (e.g. for bi-manual interaction). The capacitive sensing prototype uses input from 4 different sensors arranged around the screen.

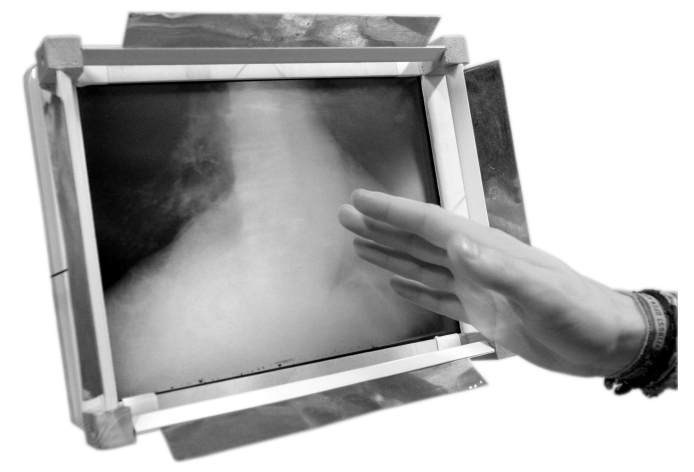

Figure 1. The picture shows a prototype of a screen equipped with a Thracker device. In this example the user navigates (scrolling/zooming) through an X-ray photograph by moving the hand and performing gestures in front of a screen.

The paper is structured as follows. In Section 2.1, a short overview on the backgrounds of capacitive sensing is given. Related work from science and arts is discussed in Section 2.2. The fully working prototype, comprising sensing capabilities and demonstration applications is presented in Section 3. Application potentials for the Thracker are shown in Section 4. To verify our approach, we conducted a small user study which is reported on in Section 5. We conclude our paper by summarizing our findings and outlining future work in Section 6. 


\section{Capacitive sensing}

In this section we first outline the basic concept of capacitive sensing and review relevant research. Even though capacitive sensing has evolved since the first Theremin, we show the potential of this interaction technique which is not yet utilized in human computer interaction.

\subsection{Basic principle of capacitive sensing}

The simplest capacitor consists of two metal plates put close together without touching each other. When current is placed on those plates they can store energy. When the current is removed and the plates are connected through a circuit, the stored energy initiates a current. Thus, a capacitor works like a small accumulator. The capacity (capacitance) depends on the size of the plates and their distance.

Using the effect mentioned above, one can measure and track the distance between a sensor and an object. For this, one of the two plates of a capacitor is replaced by the object to be tracked. In order to hold enough free electrons or charged molecules, the object has to have a relatively high dielectric constant. Most of these materials are electrically conductive like metal, water or the human body. When the object gets closer to the plate, the capacitance of this pseudocapacitor increases. One can measure the capacitance of this capacitor and from this estimate the distance between sensor plate and object. Connecting the object or person to ground can increase the availability of free electrons in it and thus the sensitivity of the device.

The most common way to measure the capacitance of a capacitor is to use a resonant circuit. Depending on the capacitor's capacitance, the resonant circuit resonates faster or slower. This technique of measuring distances between a sensor and an object is called capacitive sensing. Such sensors allow measurement of microscopic displacements in the range of micrometers. They are the industry standard for ultra-high precision measurements in many application areas.

However, capacitive sensing can also be used to track objects, e.g. the human hand as electrically conductive object, in larger ranges. The feasibility of using capacitive sensing for position and gesture input to enable intuitive humancomputer interaction is the main contribution of this work.

\subsection{Related work}

Capacitive sensing for gesture interfaces is not a totally new idea. Artists have used Theremins as input devices for video installations or light shows:

One of the first capacitive sensing interfaces was a musical instrument called Theremin, which was invented in 1919. The music instrument player can adjust volume and pitch by changing the distances between his hands and two antennas. In such an arrangement, the sensing is relative and due to the feedback (the music created) the absolute position is of minor importance. Changes in the environment and in the system are automatically compensated by the artist as he or she considers the tone rather than the absolute position of the hand as the relevant parameter. Making music in the air using novel technologies, away from traditional instruments, is quite common for performance artists. Recently, the Austrian artist Neugebauer used a similar technology for his project Soundcage in [5].

An extensive discussion of physical interfaces in arts in general is given by Bongers in [1]. This work also discusses capacitive sensing as input modality. Here, too, using capacitive sensing as relative input with a direct feedback removes many problems faced when creating more generic human computer interfaces.

The technology of capacitive sensing itself is already part of today's computers, e.g. in the touchpads of current laptops. There, input is limited to a very small range of sensor to hand. We extend the sensing range to explore the impacts on the way input to a system can be generated. However, using capacitive sensing for medium-scale position tracking has not been pursued so far. This might be due to the inherent hardware limitations mentioned in Section 3.4.

Smith et Al. [7] and Zimmerman et Al.[8] explored the potentials of electric field sensing as input modality. They e.g. developed contactless hand tracking devices using electric field sensing. This technique uses sensor plates which produce an electric field and measure its disturbance by the human hand. Electric field sensing yields higher resolution than capacitive sensing but requires significantly more hardware and processing.

Jacky Lee et al. [4] developed a 3D interface device for CAD workstations which uses capacitive sensing. This device (iSphere) only measures three different states (distant, close, pressure). The user needs to touch the iSphere for interaction. Interaction at a distance is not supported.

Different commercial integrated circuits (IC) are available that are based on capacitive sensing, e.g. [6]. These ICs are targeted at touch control applications. Ethertouch [3] is a recently developed capacitive sensing IC which provides 12 channels for sensors and was engineered for higher precision than Thracker. No commercial applications in HCI have been presented so far.

Overall in human computer interacting there are very few results discussed that make use of capacitive sensing on a larger scale. The support for gesture input using several sensing plates, as introduced in this paper, is to our knowledge new. 


\section{Prototype}

We built a prototype system that allows 2D gesture recognition based on capacitive sensing. The prototype is attached to a tablet PC with a 12.1" screen. Four sensor plates are arranged around the screen as shown in Figure 1. They are connected to the Thracker device on the back of the tablet PC via USB.

\subsection{Thracker hardware}

The Thracker device is a circuit board containing four separate equal sensing modules (Figure 2) - one for each sensor plate. They share a common $5 \mathrm{~V}$ power supply and ground from the USB port and a clock signal generated by a NE555 timer IC. Each module has one sensor plate connector and eight binary outputs which connect to one of the four 8-bit-ports on an IOWarrior40 USB interface chip [2].

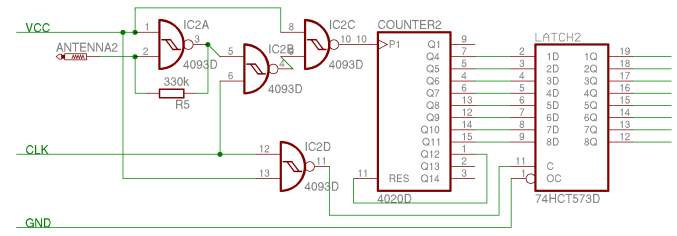

Figure 2. Electrical circuit for one sensor module of the Thracker device. The current prototype uses four modules which are connected to sensor plates on each side of the computer screen.

A NAND gate, a $300 \mathrm{kOhm}$ resistor and a sensor plate in each module provide a rough resonant circuit. When a hand approaches the sensor plate, the capacitance of the sensor plate increases resulting in a lower frequency of the resonant circuit. It usually resonates at $60 \mathrm{kHz}-120 \mathrm{kHz}$ depending on the distance of the hand.

This signal is fed into a 14-bit binary ripple counter. A clock signal controls whether the signal from the resonant circuit reaches the counter. This is needed to assure that the value of the counter does not change while copying it into the latch.

Every time the input P1 on the counter IC changes (60.000 - 120.000 times a second) it counts up by one. As the USB-IO-chip has only 32 inputs ( 8 for each module), not all of the 14 bits the counter provides can be read out. Thus only bit $4-11$ of the counter are connected to the latch. Bit 12 is connected to the reset pin. The latch assures that the bit pattern the IO-chip receives does not change during readout. Every $30 \mathrm{~ms}$, the latch IC copies the input bits (1D-8D) to the output bits (1Q-8Q). The input bits do not change during the time in which the latch copies them as the controlling clock signal disconnects the resonance circuit from the counter for this timespan. As the clock signal only switches to zero for a very short time, most of the time the counter counts. This maximizes the resolution of the circuit. The content of the latch is read out by the USB-IO-chip and sent to the host computer for processing.

\subsection{Thracker software}

The raw values sent from the IOWarrior40 chip are processed by a Java Application using a standard API. As the counter IC overflows every $35-80 \mathrm{~ms}$, the Thracker device has to be polled at least 30 times per second. The raw values are filtered for obviously invalid values and averaged to remove jitter. From this data the software calculates the distance of the hand with regard to each of the sensing plates using hard-coded reference values. We tested an algorithm which uses manual calibration data to calculate a distance function. However, this has not yielded significant improvements in precision.

\subsection{Advantages of Thracker}

Capacitive sensing in general has advantages over optical, acoustical or radio-based tracking methods. In the following we highlight issues that go beyond what was discussed in related work.

\subsubsection{Low cost sensors}

Sensors can be built at vary low cost. As only some standard ICs and a commonly available USB interface chip are used in Thracker, the total cost of the prototype (excluding circuit board) was under 15 Euro, mainly for the components realizing the USB-connection. When integrating the concept of capacitive sensing into the device (e.g. an appliances or a tablet PC) the cost for the hardware can in many cases be neglected as it will be only cents.

\subsubsection{Small size, robustness and invisibility}

Capacitive sensors can be built in a very small form factor. When larger distances (dozens of centimeters) have to be measured, bigger sensor plates may be necessary. However, those can be very flat. The sensor form can be adjusted to fit certain requirements. The Thracker device is about the size of a cigarette box. The sensor plates are $4 \mathrm{~cm}$ wide.

Capacitive sensing devices do not need moving parts, and can be embedded into solid cases without openings for sensors. This makes them ideally suited for areas with a high threat of vandalism and heavy duty environments. Certain devices already have metal parts that could be reused as sensors.

Capacitive sensing can be embedded into devices without showing any signs of it on the outside. This eases design 
of visually attractive interface devices. However, our prototype currently uses four not quite invisible sensor plates and a box hidden on the back of the tablet PC.

\subsubsection{Scalability, high precision and speed}

When a greater area has to be covered, additional sensors can easily be installed. Administrative overhead is relatively small. Thracker's USB interface chip has only 32 digital inputs. To add additional sensors we need to reduce the readout resolution of individual modules (e.g. eight sensors with 4 bit each). This requires only minor modifications of our hardware design. A serial protocol between sensor modules and interface chip would provide even more scalability.

Capacitive sensing allows for a precision in micrometer ranges. This is of course only possible in close proximity. But even in the range of up to 20 centimeters Thracker can sense small movements of about a centimeter. As very little processing is needed on the acquired data, capacitive sensing is very fast. Unlike optical tracking the sensors do not suffer from occluded markers or a changing light conditions in the environment. Changes in humidity or temperature do not influence the measured values significantly.

\subsubsection{Ease of use}

Once calibrated, capacitive sensing devices do not need additional care. Users interacting with them do not have to carry a transponder or optical marker. Using such a device is intuitive and in most cases no explicit training is needed.

\subsection{Limitations of Thracker}

While capacitive sensing has a lot of advantages there are some significant disadvantages. These may explain why commercial applications of capacitive sensing are rare.

\subsubsection{Sensitivity quickly decreases}

The major problem with capacitive sensing is that its resolution is highly dependent on the distance. To measure distances of over $30 \mathrm{~cm}$ larger sensor plates and highly sensitive circuits have to be built. Active sensing methods can provide greater ranges. Passive capacitive sensing seems to be unfit for tracking objects in larger areas (e.g. 30 centimeters or more).

\subsubsection{Objects interferance}

Capacitive sensors only detect the presence of electrically conductive objects. This need not be the object to be tracked but can be another object which rather should not be tracked. Even if the interfering object does not move it dampens the signals and reduces the tracking resolution. This can only partly be compensated by shielding.

Our prototypical implementation of Thracker suffered from the problem that the sensor plates had to be attached to the monitor. Unfortunately a monitor contains a big metal sheet behind the screen. This strongly dampens the signal. The detection range of Thracker sank from about $60 \mathrm{~cm}$ to $20 \mathrm{~cm}$ when attaching the sensor plates to a monitor. We compensated for this loss of precision by placing sensor plates on each side of the screen. Thus we could limit the range a sensor plate needed to cover to half a screen. All data from a plate which indicated a larger distance between hand and sensor plate was ignored for 2D tracking.

Additionally other objects passing the sensor can spoil the measurement. When an other person walks past the Thracker-equipped monitor he or she might move the cursor minimally. This makes it hard to provide reliable capacitive sensing for heavily populated environments. The indifferent recognition behavior poses a challenge when integrating capacitive sensing hardware in handheld device.

\subsubsection{Limited and ambiguous data}

The only information a capacitive sensor returns is its capacitance. A certain capacitance can result from one person standing in front of the sensor or from two persons standing a little farther away. Disambiguation can sometimes be achieved by using additional sensors and filtering.

\section{Interaction potentials}

Because Thracker measures the distance of the hand from each border, it offers additional degrees of freedom (DOFs) unique to this setup. We defined two modes which allow 3D interaction or 'Pick and Drop' interaction.

In $3 \mathrm{D}$ mode we can calculate the $\mathrm{Z}$ axis distance to the screen from the distances to two opposite plates and the distance between the plates themselves:

$$
z=d_{1} \arccos \left(\frac{w 2+d_{1}^{2}-d_{2}^{2}}{2 w d_{1}}\right)
$$

Whereas $d_{1}$ and $d_{2}$ are the distances between object and sensor plates and $w$ is the distance between the two sensor plates.

This enables the user to interact in three dimensions with the screen. However, interpretation of $\mathrm{Z}$ axis data is not always clear. If the user wants to end an interaction session he or she will pull back th ehand out of reach of the sensors. The sensors will interpret this first as a normal movement in $\mathrm{Z}$ axis before they can detect the user's intention of leaving the sensor area. If the $\mathrm{Z}$ axis data is used, e.g., for zoom control, this behavior will inadvertently change the displayed information. A workaround would be to introduce a small 
delay between gesture and display feedback. Gestures will only result in display changes if they stay within the sensor range long enough.

The $\mathrm{Z}$ axis data could also be used to simulate mouse clicks. This requires only two discrete positions. Movement farther away from the screen than three centimeters will be interpreted as pointer movement whereas movement within three centimeters of the screen will be interpreted as clicks.

The second mode which we called 'Pick and Drop' allows the user to intuitively interact with objects on the screen. The sensor plates of the Thracker device do not measure the distance to the center of the pointing hand but measure the distance to the nearest part of the hand. Thus Thracker can calculate the diameter of the pointing hand. A user can interact with the screen by spreading thumb and fingers (Figure 3).

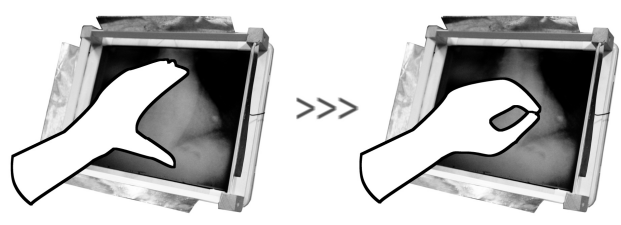

Figure 3. The user can interact with objects on the screen by making a 'picking' gesture. This interaction mode is unique to Thracker.

A possible intuitive use of hand spreading detection is an interface which uses a 'Pick-and-Drop' metaphor. The user can pick a virtual object on the screen by closing the hand, then move the object and release it by opening the hand again.

The limited resolution of our current prototype allowed reliable detection of hand spreading in the center of the screen only. Alternatively, the user could use both hands for a gesture resembling the parting of a curtain. Such bimanual interaction can be recognized with the current prototype throughout the screen.

As mentioned before, Thracker can not distinguish between small, close objects and large objects that are far away. Thus it cannot determine if the user's hand approaches the screen or is spread. Hand spreading can only be detected if the user's hand is close to the screen. While picking up a virtual object will probably be done close to the screen, the user may release the object when the hand is farther away from the screen. This cannot be distinguished from a movement in the direction of the screen. Thus only one of the interaction modes can be implemented into an application. Placing an additional, transparent sensor plate on the screen would allow for both modes to be used at a time.

Due to the limited resolution of our prototype those two modes could not be fully implemented and studied. How- ever, we integrated a way of controlling zoom by spreading the hand into our viewer application to give users the chance to explore this type of interaction. Thracker offers unique advantages not found in other human interaction devices and it allows for intuitive and quick absolute and dynamic positioning.

\subsection{Possible applications}

An obvious usage area for Thracker-equipped screens are interactive displays in museums, exhibitions or public places. Users can interact with art, underground maps or timetables in an simple and intuitive way. Thracker is resistant to vandalism, does not require people to touch the input device and is quite cheap. Existing displays can be easily equipped with a Thracker device. While large TFT displays may dampen the sensitivity of the Thracker device, rear- or front-projection displays are ideally suited.

As Thracker is low-cost, even static paper posters could be made interactive. An interactive poster could sense if someone is standing in front of it. The user could tap special areas on the poster to hear additional voice information or a music sample.

Workers who have to wear protective gloves have difficulty interacting with touchscreens or mice. Thracker enables them to interact with a computer without exposing its input devices to hazardous environments. Similarly in a sterile operating room surgeons may not touch unsterile input devices like mice. Thracker could enable them to easily pan and zoom in x-ray images by simple hand movements. Thracker could also be integrated in vandalism-proof ticket vending machines.

On a larger scale Thracker could be used to track persons in a room. On a smaller scale Thracker could be used in interactive toys, e.g. a small robot which always turn in the direction of its owner's finger.

\section{Evaluation}

We conducted an initial user study with 10 participants ( 1 female, 9 male) in the age between 22 and 40 . All participants are familiar with computers and traditional input devices and all use computers in their work. They were asked to complete two tasks and fill out a questionnaire. Every task was done two times to measure learning effects.

In the first task, the user had to center and magnify a certain feature in a large image by scrolling and zooming using their hand. Scrolling was done by moving the hand to a side of the screen. Zooming was done by putting each hand on an opposite side of the screen (simplified curtain gesture). We planned to use hand spreading for zoom control (curtain gesture) but found in a pre-test users having problems doing the right gesture in the right spot. The time 
to accomplish this task was measured.

All users managed to understand and handle scrolling at once. Zooming of an image proved to be more difficult because users tried to zoom in by moving the pointing hand to one side of the screen and concurrently putting the other hand on the opposite side. The movement of the pointing hand displaced the image and required the users to re-adjust the displayed frame. 9 out of 10 users managed to improve their speed in the second pass. While the users needed an average 46 seconds in the first pass, they completed the second pass in an average of 24 seconds.

The second task tested absolute positioning using Thracker. Twelve (four by three) numbered rectangles, each 256x256 pixels in size, filled the screen. The user had to click them in the correct order. As $\mathrm{Z}$ axis tracking was not implemented in the prototype, clicking was done by hovering over a rectangle for at least 0.8 seconds (dwell time). This task was completed very fast and easily by all users. The users' speed did not increase significantly in the second pass.

A questionnaire was used to ask the users to rate how intuitive and usable they perceived the applications. They were also asked what advantages and disadvantages they saw compared to touchpads and mice. We also wanted to know which possible application areas they saw for Thracker. Most users liked the concept and interaction using Thracker. All users found scrolling extremely intuitive and easily usable but rated zooming not very intuitive but usable. Absolute positioning on the screen as required in the second task was perceived extremely intuitive and easy to use. The possible uses mentioned were consistent with our suggestions from Section 4.1.

From the user study we conclude that absolute and dynamic positioning using gesture interfaces is reasonably intuitive and user-friendly. Additional special gestures - in this case for zooming - are less intuitive. The significant speed increase when repeating the first task indicates that zooming using gestures may not be intuitive but easily learnable. The gestures required for zooming need refinement to avoid wrong interpretation of hand movements.

\section{Conclusion and future work}

We introduced Thracker, a human interface device for gesture recognition which allows low-cost, robust and contact-less tracking of a human hand. Thracker enables the user to interact in 3D space with a display. The 'Pick and Drop' mode provides new interaction possibilities. We found that Thracker enables users to intuitively and easily navigate large images. Absolute and dynamic positioning seems to be intuitive even when using new gesture interfaces. It's probable that users translated their scrolling and pointing experience with a mouse to the new interaction technique. A lack of similar experiences for gestures is obvious. While mouse gestures are available in web browsers they seem to be neither intuitive nor commonly used enough to aid people in using touchless gesture interfaces. Research of universal interaction gestures might enhance interaction with gesture interfaces.

From our experience with implementing the prototype, we see room for hardware improvement. The Thracker device is built from standard components and has not been refined much. It has been found that a resonant circuit might not be the best means to measure capacitance. Noise and fluctuations in measurement could probably be minimized using specialized ICs.

Further optimization has to go into hardware design to improve the sensor resolution. A prototype which uses a rear-projection display could provide higher resolution. This would enable us to test the new interaction techniques we found with a larger user base.

\section{Acknowledgements}

The work has been conducted in the context of the research project Embedded Interaction ('Eingebettete Interaktion') and was funded by the DFG ('Deutsche Forschungsgemeinschaft').

\section{References}

[1] B. Bongers. Physical interfaces in the electronic arts. Trends in Gestural Control of Music, January 2000.

[2] Codemercs Inc. IO Warrior USB I/O Chip. http: / / www. codemercs.com/iowarriore.html, visited January 2006.

[3] Ethertouch Inc. Ethertouch. http: //www. ethertouch. $\mathrm{com} /$, visited January 2006.

[4] C.-H. Lee and T. S. Yuchang Hu. iSphere: a Proximity-based 3D Input Interface. CAAD Futures, 2005.

[5] C. Neugebauer. SOUNDCAGE - Entwicklung und praktische Ausf"uhrung eines MIDI-Eingabeger"ates in Form eines Laser-Klangk"afigs (available in German only). Diploma Thesis, Universit"at f"ur K"unstlerische und industrielle Gestaltung Linz, Austria, 2000.

[6] QProx Inc. QT110 Chip Family. http://www. qprox. com, visited January 2006.

[7] J. Smith, T. White, C. Dodge, J. Paradiso, N. Gershenfeld, and D. Allport. Electric field sensing for graphical interfaces. IEEE Comput. Graph. Appl., 18(3):54-60, 1998.

[8] T. G. Zimmerman, J. R. Smith, J. A. Paradiso, D. Allport, and N. Gershenfeld. Applying electric field sensing to humancomputer interfaces. In CHI '95: Proceedings of the SIGCHI conference on Human factors in computing systems, pages 280-287, New York, NY, USA, 1995. ACM Press/AddisonWesley Publishing Co. 